\title{
Squamous Cell Carcinoma Lung Presenting As A Painful Thigh Mass : A Case Report
}

\author{
Syed Besina, M.D., Othman Salim Akhtar, M.B., Nazia Bhat, M.B., Tazeen Jeelani Samoon, M.B., \\ Department of Pathology, SKIMS, Soura, Srinagar
}

\section{A B S T RA C T}

A 75 year old male presented with a painful swelling in the left thigh. Fine needle aspiration biopsy performed revealed the presence of a Squamous cell carcinoma, prompting further evaluation. This led to the identification of an upper zone mass in the left lung which was excised; intra-operative squash cytology revealing the presence of a Squamous cell carcinoma. The patient then underwent a complete excision of the thigh swelling. Three months after the surgery, the patient died of a stroke. We report this case on account of its rarity and to highlight the role of intra-operative squash cytology in establishing the diagnosis of such lesions. JMS 2014; 17(2):71-73

Key Words: Lung Pathology; Lung cancer surgery; Intraoperative squash cytology; skeletal muscle metastasis; thigh mass

\section{INTRODUCTION}

Lung Cancer is the leading cause of cancer related deaths globally. Most patients with lung cancer are symptomatic at presentation and present with respiratory symptoms such as cough, dyspnea or bloody sputum, with systemic symptoms, or with symptoms related to distant metastasis. ${ }^{1}$

More than $20 \%$ of patients have distant metastasis at the time of diagnosis. Brain, bone, liver and the adrenals are the most common sites of metastasis. ${ }^{2}$ Metastases to skeletal muscle are uncommon and few cases have been reported in the literature. Only rarely are these the first clinical manifestation of primary tumor. ${ }^{3}$

Here we report a case of a non-small cell lung cancer, presenting as a large painful swelling of the left thigh, further evaluation of which led to identification of the primary.

\section{CASE REPORT}

A 75 year old male presented with a two month history of pain and swelling of the left thigh. There were no other symptoms. General physical, chest and abdominal examinations were unremarkable. Local examination of the

\section{Correspondence}

Dr. Syed Besina Yasin, M.D.

Addl. Professor, Department of Pathology,

SKIMS, Srinagar

Email: drsyedbesina@yahoo.com left thigh revealed a large, $10 \times 15 \times 4 \mathrm{~cm}$ swelling on the posterior aspect, just below the gluteal fold, with normal overlying skin. The swelling was non tender, partly mobile and no pulsation or bruit was felt. Chest and abdominal examinations were unremarkable. A clinical diagnosis of a soft tissue tumour was made.

Magnetic Resonance Imaging (MRI) showed a peripherally enhancing cystic mass lesion, $12 \times 0.8 \times 0.8 \mathrm{cms}$, in the postero-medial compartment muscles of the left thigh with a possible neoplastic etiology [Figure 1]. Fine Needle Aspiration Cytology (FNAC) of the swelling revealed degenerated malignant epithelial cells with Squamous differentiation, suggestive of a Squamous Cell Carcinoma [Figure 2].
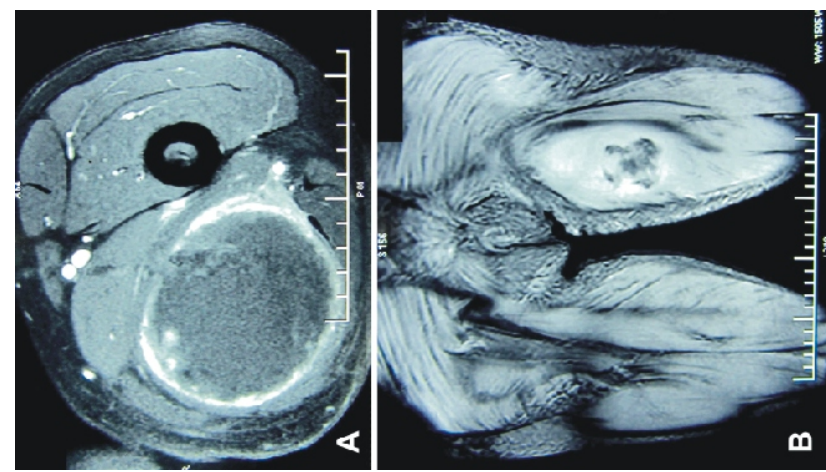

Figure 1: (A) A T2 Weighted Axial MR Image shows a cystic mass with a thick ragged enhancing wall within the postero-medial compartment muscles the intramuscular location of the lesion (B) T2 weighted Saggital MR Image of the same mass. 


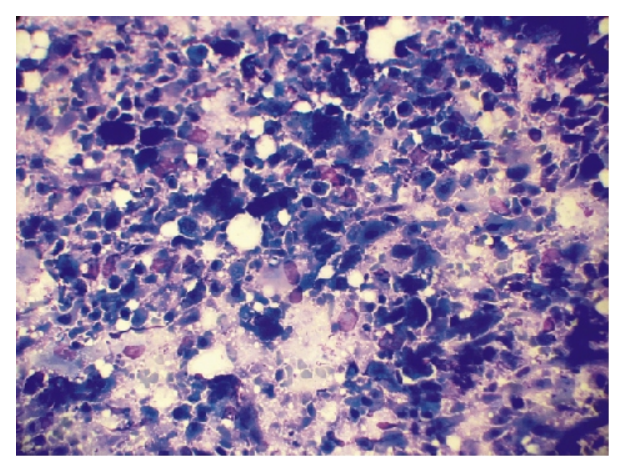

Figure 2: Fine Needle Aspiration Cytology of the thigh mass showing malignant epithelial cells with features suggestive of a Squamous cell carcinoma (MGG, High Power x40)

The patient was referred to our institution for review, where he was further evaluated. His routine blood investigations including a hemogram, liver \& renal function tests, serum calcium all were within normal limits. A chest X-Ray, however, revealed a left upper zone mass. Contrast Enhanced Computerized Tomography (CECT) of chest and abdomen confirmed a focal lesion in the peripheral aspect of the left upper lobe suggestive of a neoplastic etiology [Figure 3]. No nodal or liver metastases were found. A biopsy under radiological guidance from the lung lesion failed to obtain sufficient tissue for diagnosis. A posterolateral thoracotomy was performed and intra-operative squash cytology from the lesion confirmed the presence of a Squamous cell carcinoma. A left upper lobectomy was then performed. As the investigations had revealed no metastasis elsewhere, the operating surgeon resected the mass lesion in the thigh as well. Histological examination of the lobectomy specimen confirmed the diagnosis of a moderately differentiated Squamous cell carcinoma resection margins were free [Figure 4]. A similar tumor was seen infiltrating the muscle in the resected mass lesion from the thigh, with normal overlying skin; again the resection margins were free.

The patient died of a stroke three months after the surgery.

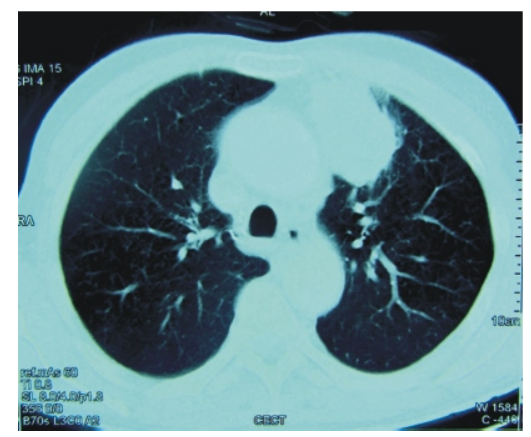

Figure 3: An axial section of a CECT thorax reveals a focal lesion in the peripheral aspect of the left upper lobe.

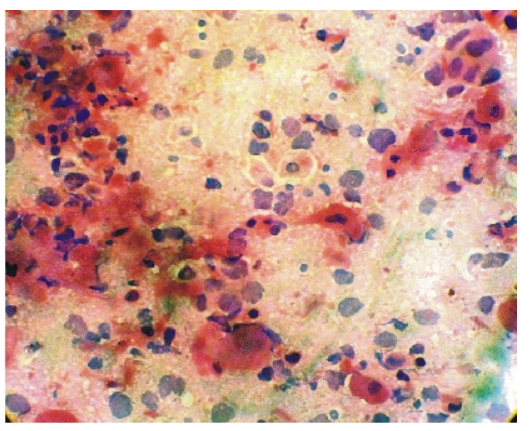

Figure 4: Intra-operative Squash Cytology shows numerous malignant epithelial cells suggestive of a Squamous cell carcinoma. (H\&E, High Power x40)

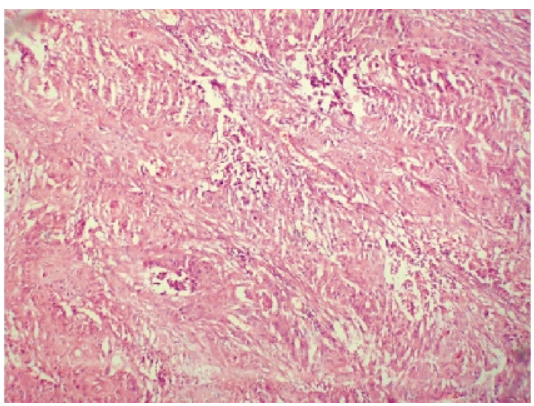

Figure 5: Histology of the lung lesion showing sheets of cells with abundant eosinophilic cytoplasm and numerous dyskeratotic cells, suggestive of a Squamous Cell Carcinoma (Low Power x10)

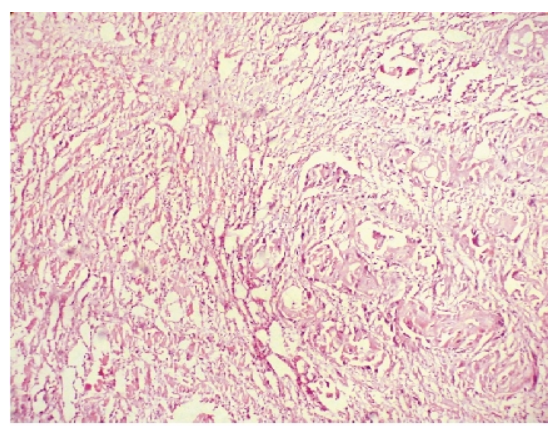

Figure 6: Histology of the thigh mass showing malignant epithelial cells in nests, infiltrating the surrounding muscle. (Low Power x10)

\section{DISCUSSION}

Sixty two cases of skeletal muscle metastasis from NonSmall Cell Lung Cancer have been reported in the literature so far. Metastatic lesions were the initial manifestation of the disease in 48 (78\%) of these cases. The actual incidence of metastasis to skeletal muscle is however, not known. Skeletal muscles are thus remarkably resistant to distant metastasis, despite constituting more than $50 \%$ of total body weight and being highly vascular. Several theories have been put forward to explain this phenomenon and suggest a combination of factors such as muscle contraction, $\mathrm{pH}$ and 
lactic acid, amongst others as being responsible. ${ }^{4}$

The majority of skeletal muscle metastases are from epithelial tumors, most frequently Squamous Cell Carcinoma of the lung. Other primaries include renal cell carcinoma, leukemia, lymphomas and anaplastic carcinomas. $^{3}$

Like in our patient, the most frequent presentation of such lesions is pain with or without swelling. ${ }^{5}$

Diagnosis remains tricky especially in the absence of a known primary. On Computerized Tomography (CT) lesions appear as rim enhancing masses with central hypoattenuation mimicking intramuscular abscesses. ${ }^{6}$ Even as MRI is the modality of choice for characterizing soft tissue lesions, the features of skeletal muscle metastasis are nonspecific, showing increased signal intensity relative to skeletal muscle on T2 weighted images and reduced signal intensity on T1 weighted images. On gadolinum administration, they show heterogeneous enhancement. ${ }^{7}$

Fluorodeoxyglucose (FDG) positron emission tomography (PET) -CT is emerging as a sensitive tool in the recognition of distant metastasis with the exception of the brain. ${ }^{8}$ However, in muscle, an injection site inflammation may show increased FDG uptake and be confused for a neoplastic lesion.

This underlines the importance of histologic diagnosis. A fine needle aspiration biopsy can be obtained with or without the help of radiological guidance. Alternatively, an excision biopsy can be attempted. As in our case, where there is a concomitant lung lesion, intra-operative squash cytology can provide a rapid diagnosis and confirm the presence of the tumor. The accuracy of touch imprint cytology in diagnosing lung lesions is well established. ${ }^{9}$

Because there are no large series in literature, the management of skeletal muscle metastasis is not well defined. Treatment may include palliative radiotherapy to the site to reduce pain and swelling when present. Surgical excision, as in our patient, may be attempted in absence of metastasis elsewhere. ${ }^{10}$

Skeletal muscle metastasis usually portends a poor prognosis. Most patients die, with a median survival of less than 6 months. ${ }^{5}$

Our patient presented with a painful swelling in the left thigh which was clinically diagnosed as a soft tissue tumor. FNAC showed the presence of Squamous cell carcinoma and led to further evaluation which revealed the presence of a lesion in the left lung on X-Ray. While the lesion was further characterized on CECT, it was only intra-operative squash cytology, later confirmed by histology that led to the diagnosis of metastatic Squamous cell carcinoma of lung.

Our case underlines the importance of considering a metastatic neoplasm in the differential diagnosis of a painful intramuscular swelling. In addition it highlights the important role cytology and histology play in establishing a definitive diagnosis.

\section{REFERENCES}

1. G. Buccheri, D. Ferrigno. Lung cancer: clinical presentation and specialist referral time. Eur Respir $J$ 2004; 24:898-904

2. Onuibgo WIB. Patterns in metastasis in lung cancer: A review. Cancer Res 1961;21:1077-1085

3. Di Giorgio A, Sammartino P, Cardini CL et al. Lung Cancer and Skeletal Muscle Metastases. Ann Thorac Surg 2004; 78:709-11

4. Seely S. Possible reasons for the high resistance of muscle to cancer. Med Hypotheses 1980; 6:133-137

5. Pop D, Ahmad SN, Venissac N, Patrice G, Otto J, Poudenx M, Moroux J. Skeletal Muscle Metastasis from Non-small Cell Lung Cancer. $J$ Thorac Oncol 2009; 4:1236-1241

6. Pretorius ES, Fishman EK. Helical CT of Skeletal Muscle Metastases from Primary Carcinomas. AJR 2000; 174:401-404

7. Williams JB, Youngberg RA, Bui-Mansfield LT, Pitcher JD. MR Imaging of Skeletal Muscle Metastasis. AJR 1997; 168:555-557

8. Emmering J, Vogel WV, Stokkel MP. Intramuscular metastases on FDG PET-CT: a review of the literature. Nucl Med Commun 2012, 33:117-20

9. Paulose RR, Shee CD, Abdelhadi IA, Khan MK. Accuracy of touch imprint cytology in diagnosing lung cancer. Cytopathology 2004; 15:109-112

10. Mogi A, Takayuki K, Yamaki Ei, Kuwano H. Successful Resection of Stage IV Non-small Cell Lung Cancer with Muscle Metastasis as the Initial Manifestation: A Case Report. Ann Thorac Cardiovasc Surg 2012; 18:468-71 [Epub] 